\title{
Simple and Reliable Harmonic Mitigation Strategy in Power Electronic Converter for Grid Integration of Renewable Energy Resources
}

\author{
Ngoc Bao Lai ${ }^{1}$ and Kyeong-Hwa Kim ${ }^{2}$ \\ ${ }^{1,2}$ Department of Electrical and Information Engineering \\ Seoul National University of Science and Technology \\ 232 Gongneung-ro, Nowon-gu, Seoul, 01811, Korea \\ ${ }^{2}$ Corresponding Author \\ k2h1@seoultech.ac.kr
}

\begin{abstract}
A new simple and effective scheme to improve the power quality of grid-tied inverters in distributed generation $(D G)$ system under faulty grid conditions is presented. In order to regulate the fundamental and harmonic components effectively with low computational burden, the proposed control scheme is constructed with two controllers, namely, a proportional-integral controller and a high bandwidth proportional harmonic controller. The former is in charge of injecting the fundamental positive-sequence current into the grid. The latter is designed to alleviate the harmful effect due to abnormal grid voltage. Since the latter controller is specially used to improve the performance of system in high frequency ranges, it is desirable to separate the high frequency harmonics from the grid voltage and current. To obtain the harmonic components, a fourth-order band-pass filter is employed. Then, the undesirable harmonic components can be completely suppressed by proportional decoupling controller, while the exchange powers are exclusively controlled by proportional-integral (PI) decoupling controller. Simulations and experimental results are presented to verify the simplicity and effectiveness of the proposed scheme.
\end{abstract}

Keywords: Harmonic mitigation, Low computational burden, Microgrid, Power converter, Renewable energy, Robustness enhancement

\section{Introduction}

The steady growth in electricity demand and the depletion of fossil energy have been creating an increased interest in the renewable energy resources and construction of microgrid. Moreover, to accelerate the economy and stability of operating power systems, the injected renewable energy into the grid by means of the distributed generation (DG) system has been shooting up in the last decade [1]. One of the important requirements imposed by all power system operators regarding grid-tied systems such as the grid-tied inverter for photovoltaic and wind turbine applications is the quality of distributed power. In order to preserve the power quality, the total harmonic distortion (THD) in injected current needs to be met certain standards such as IEEE-519 or IEC 61000-3-2, which has been enacted for the purpose of satisfying the gird integration codes [2], [3].

The proportional-integral (PI) decoupling control with the grid voltage feedforward is commonly used for current-controlled inverters because of its simplicity and stability [4]. However, the conventional PI decoupling regulator does not give proper performance under distorted and unbalanced grid voltages due to the limitation of control bandwidth. To improve the power quality of DG systems, several research works on the harmonic compensation strategy have been investigated. The harmonic compensation methods are 
classified as two groups, namely, selective, and non-selective scheme. One of the most widely used methods as selective harmonic compensation scheme for grid-tied inverters is proportional resonant (PR) controllers [5], [6]. These controllers are designed not only to regulate the fundamental current, but also to compensate harmonic terms selectively. The PR controller is known as a flexible harmonic compensation method which is highly robust against the grid disturbances. However, despite having aforementioned advantages, the PR controller reveals a quite complex structure, which may not be applicable for many commercial power applications.

As an alternative solution, non-selective harmonic compensation strategies such as the predictive control [7], repetitive control [8]-[10], and sliding mode control (SMC) [11][13] have been reported. The predictive control has been developed to minimize the forecast error in order that the reference current can be tracked properly without any errors, which yields a satisfactory current response with minimum THD level. However, the predictive current control is very sensitive to the parameter changes as a consequence of the mathematical based approach. Unlike the predictive control, the repetitive feedback control has been derived from the concept of iterative leaning control. Therefore, this control provides robust performance against periodic disturbance and ensures a zero steady-state error at all harmonic frequencies. However, the learning process often results in an unacceptable slow dynamic response. To overcome the previously mentioned difficulties regarding the predictive and repetitive controls, the SMC has been employed for grid-tied inverters [11]. The SMC has a variable structure that contains a switching term and a sliding surface representing the reference trajectory. The switching control input which takes the form of on-off control or hysteresis control is the key element of implementing the SMC. The idea behind the switching control input is that a strong control action can be generated whenever the system output deviates from the designed trajectory [12], [13]. Nevertheless, in some cases, it is difficult to design a SMC to exhibit both suitable transient performance and zero steady-state error. Moreover, the SMC suffers from its inherent chatting problem.

The existing harmonic compensation methods have their own advantages in view of control performances. However, these methods require either lots of computational capacity or high level of mathematical background for controller design due to the complexity of their control structures.

This paper proposes a simple and reliable harmonic mitigation strategy in power electronic converter for grid integration of renewable energy resources. The proposed control strategy is based on the fact that the distorted grid voltages can be expressed as the fundamental and harmonic components. Therefore, the current controllers can be designed independently in order to achieve the best control performance of each component. Due to the simplicity in control structure of the proposed scheme, the computation burden is significantly reduced, while providing a fast dynamic response and robustness against the distorted grid voltages. To verify the effectiveness of the proposed scheme, a $2 \mathrm{kVA}$ laboratory prototype grid-tied inverter incorporated with a digital signal processor TMS320F28335 has been constructed [14]. The simulations and experimental results in various operation conditions are given to prove the performance of the proposed scheme.

\section{Modeling of a Grid-tied Inverter}

Figure 1(a) illustrates the block diagram of three-phase grid-tied inverter for DG applications. In general, the grid-tied inverter consists of three main components which are a large DC-link capacitor, a power module, and an output filter to eliminate the undesirable harminic components in injected current. The injected current to utility grid is commonly controlled by a current controller through a modulator. To give an insight into the machanism of power stage, Figure 1(b) shows the simplified equivalent circuits of a 
grid-tied inverter in the synchronous reference frame. Applying the Kirchhoff's law in these simplified circuits, the voltage equations of a grid-tied inverter in the synchronous reference frame can be derived as follows:

$$
\begin{aligned}
& v_{q}=R i_{q}+L \frac{d i_{q}}{d t}+\omega L i_{d}+e_{q} \\
& v_{d}=R i_{d}+L \frac{d i_{d}}{d t}-\omega L i_{q}+e_{d}
\end{aligned}
$$

where $v_{q}$ and $v_{d}$ are the inverter output voltages in $q$-axis and $d$-axis, respectively, $i_{q}$ and $i_{d}$ are the inverter output currents in $q$-axis and $d$-axis, respectively, $e_{q}$ and $e_{d}$ are the grid voltages at the point of common coupling in $q$-axis and $d$-axis, respectively, $\omega$ is the angular frequency of grid voltage, $L$ is the output filter inductance, and $R$ is the output filter resistance.

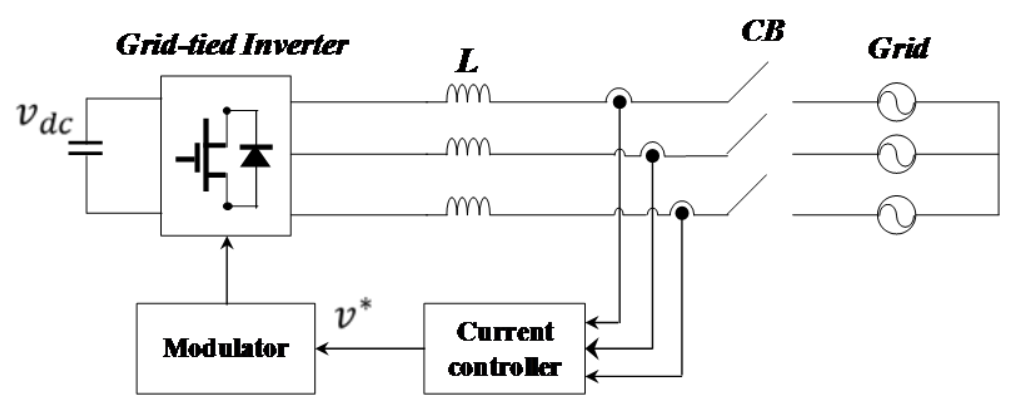

(A) Three-phase grid-tied inverter for DG

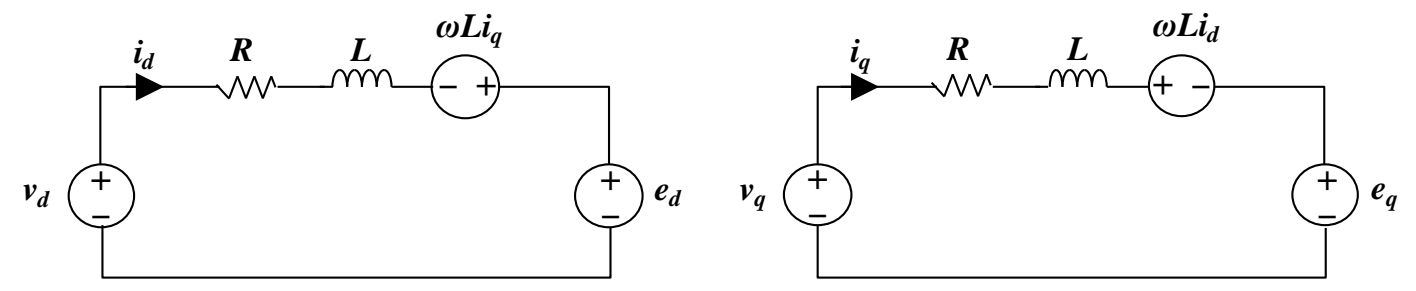

(B) d- and q-axes equivalent circuits

\section{Figure 1. Block Diagram of Three-phase DG Inverter and the Simplified Equivalent Circuits in the Synchronous Reference Frame}

\section{Proposed Harmonic Compensation Scheme}

Figure 2 illustrates the control block diagram of the proposed harmonic compensation scheme. The overall control scheme comprises a current controller, a harmonic detector, and a space vector pulse width modulator (SVPWM). Since the dynamics of primary sources are much slower than those of the inverter, it is reasonable to assume that the DC voltage is a constant value. For the purpose of controlling the fundamental and harmonic current components at the same time, the proposed current controller consists of a conventional PI decoupling controller and a high bandwidth proportional compensator. The central idea of the proposed current controller is based on the superposition principle. According to the superposition principle, the given linear system in (1) and (2) can be 
separated as two individual models, namely, the fundamental and harmonic models. Consequently, the controllers can be independently designed to archive the best performance of each system. To accomplish the superposition principle, the band-pass filters (BPFs) are employed to decompose $v, i$, and $e$ into the fundamental and harmonic variables as $V+v_{h}, I+i_{h}$, and $E+e_{q}$, accordingly, where the subscript $h$ denotes the harmonics terms and the subscript $q$ and $d$ are intentionally omitted for convenience.

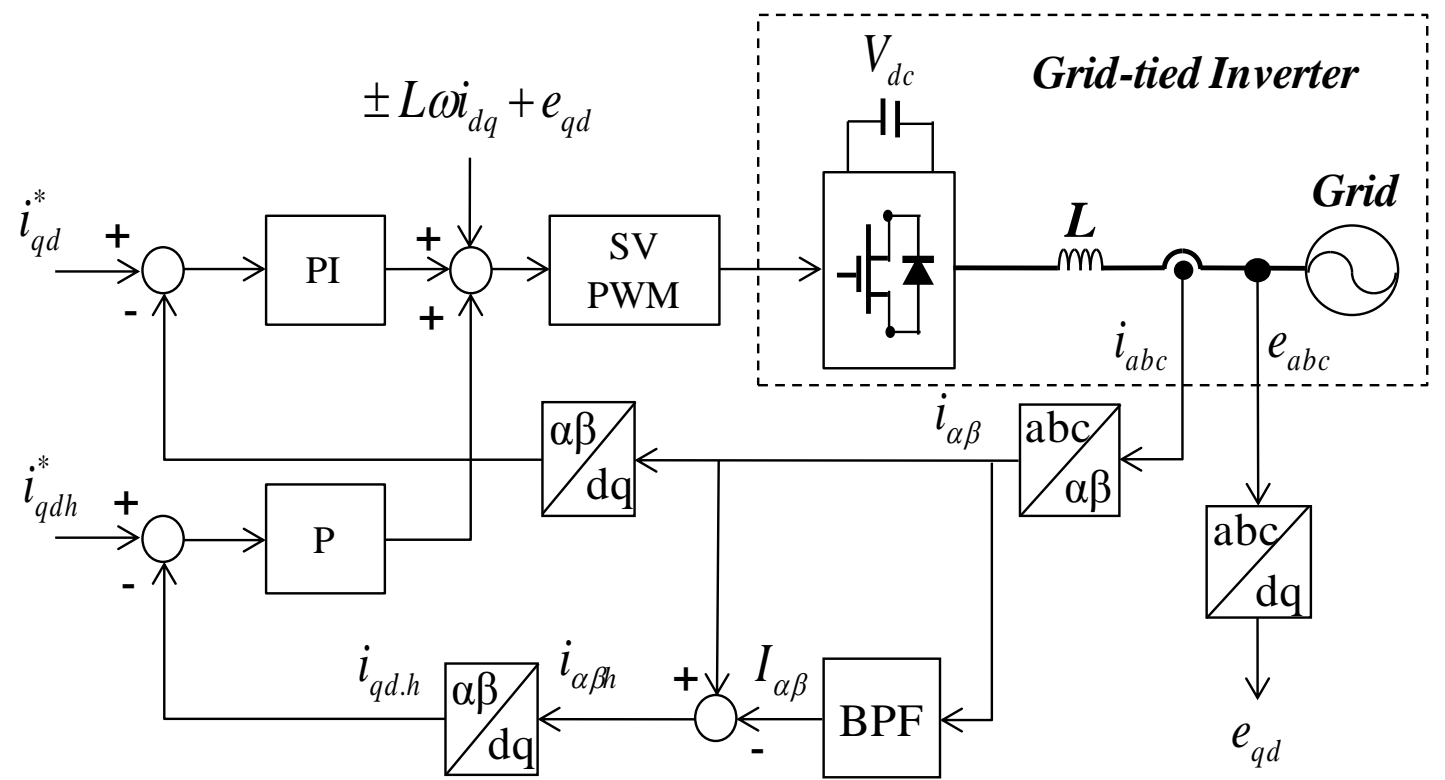

Figure 2. The Control Block Diagram of the Proposed Harmonic Compensation Scheme

Once the fundamental and harmonic signals are separated, the voltage equations of the grid-tied inverter with respect to the fundamental frequency can be expressed as follows:

$$
\begin{aligned}
& V_{q}=R I_{q}+L \frac{d I_{q}}{d t}+\omega L I_{d}+E_{q} \\
& V_{d}=R I_{d}+L \frac{d I_{d}}{d t}-\omega L I_{q}+E_{d} .
\end{aligned}
$$

As can be observed from (3) and (4), all the variables in the fundamental components have DC quantities in the synchronous reference frame. Thus, the reference currents also take the form of step signals in this frame. Bearing that in mind, a PI decoupling controller with grid voltage feedforward is used to design the controller using the fundamental model since this controller can provide excellent tracking performance for step reference input even under the parameter variations. The control law for the fundamental terms is given by

$$
\begin{aligned}
& V_{q}^{*}=\left(K_{P f}+\frac{K_{I f}}{s}\right) \cdot\left(I_{q}^{*}-I_{q}\right)+\omega L I_{d}+E_{q} \\
& V_{d}^{*}=\left(K_{P f}+\frac{K_{I f}}{s}\right) \cdot\left(I_{d}^{*}-I_{d}\right)-\omega L I_{q}+E_{d}
\end{aligned}
$$


where $s$ is the Laplace operator, $K_{P f}$ and $K_{I f}$ are the proportional and integral controller gains, respectively, $I_{q s}^{*}$ and $I_{d s}^{*}$ are $q$-axis and $d$-axis reference currents, respectively, and $V_{q}^{*}$ and $V_{d}^{*}$ are the reference voltages of the inverter, respectively.

Assuming that there is no deformation on the reference voltages, i.e. the inverter output voltages are exactly the same with the reference voltages, substituting (5) and (6) into (3) and (4) yields the transfer function of system with respect to the fundamental component as

$$
G_{f}(s)=\frac{I(s)}{I^{*}(s)}=\frac{K_{P f} s+K_{I f}}{L s^{2}+\left(R_{s}+K_{P f}\right) s+K_{I f}} .
$$

It is worth mentioning that the grid impedance value varies according to grid conditions rather than a constant parameter. Fortunately, the boundary of the grid impedance can be easily determined in order that the PI controller can be designed to cope with these variations. In this study, the controller parameters are obtained in frequency domain by choosing a proper set of the critical frequency and gain margin to ensure desirable tracking performance and system stability. The frequency response for the closed-loop system of the fundamental controller is given in Figure 3. As can be seen from Figure 3, the closed-loop system exhibits a unit gain in the low frequency regions, which proves the good tracking performance of the designed controller.

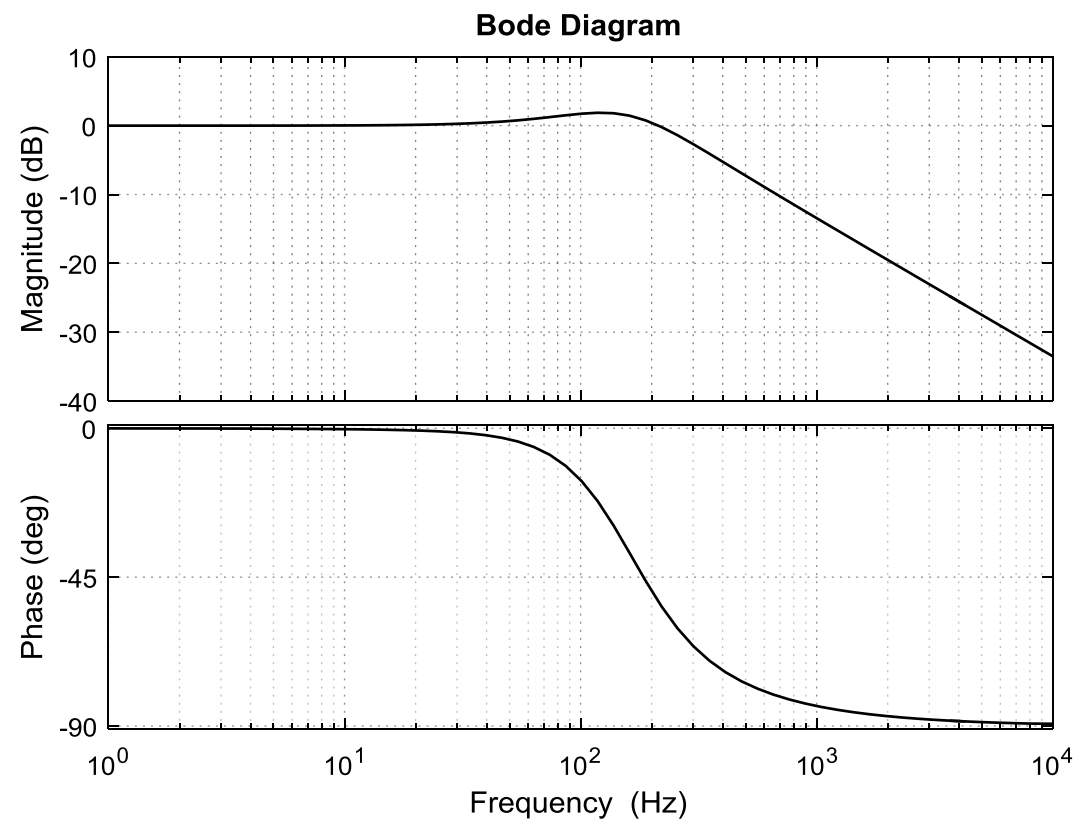

Figure 3. Frequency Response of a Grid-tied Inverter with the PI Decoupling Controller

As far as the harmonic attenuation capability is concerned, the voltage equations with respect to harmonic components can be obtained from (1) and (2) as follows:

$$
v_{q h}=R i_{q h}+L \frac{d i_{q h}}{d t}+\omega L i_{d h}+e_{q h}
$$




$$
v_{d h}=R i_{d h}+L \frac{d i_{d h}}{d t}-\omega L i_{q h}+e_{d h}
$$

To suppress the influence caused by the grid voltage harmonics, a proportional decoupling controller with grid voltage feedforward is employed using the harmonic model. The control law is given as

$$
\begin{aligned}
& v_{q h}^{*}=K_{P h} \cdot\left(i_{q h}^{*}-i_{q h}\right)+\omega L i_{d h}+e_{q h} \\
& v_{d h}^{*}=K_{P h} \cdot\left(i_{q h}^{*}-i_{q h}\right)-\omega L i_{q h}+e_{d h}
\end{aligned}
$$

where $K_{P h}$ is the controller gain, $i_{q h}^{*}$ and $i_{d h}^{*}$ are $q$-axis and $d$-axis harmonic reference currents, respectively, and $v_{q h}^{*}$ and $v_{d h}^{*}$ are $q$-axis and $d$-axis inverter reference voltages of the harmonic compensator, respectively.

With the same assumption used to obtain the transfer function of the fundamental model, the transfer function of the harmonic model can be derived by substituting (10) and (11) into (8) and (9) as

$$
G_{h}(s)=\frac{K_{P}}{L s+\left(K_{P}+R\right)} .
$$

As stated earlier, the main purpose of introducing a proportional controller for current control is to suppress the harmonics which are generally more than three times fundamental frequency. The most instructive way to reduce the effect of grid harmonics is to increase the control bandwidth of current controller. For the conventional PI decoupling controller, however, increasing the control bandwidth usually leads to the instability problems. Fortunately, by using the decomposition model, the bandwidth of the proportional controller can be archived high enough to regulate the harmonics without violating stability criteria. Through the design techniques in the frequency domain, a proper proportional harmonic compensator that is able to provide sufficient bandwidth as depicted in Figure 4 can be easily obtained. The frequency response of the closed-loop system reveals that the cutoff frequency of system with harmonic compensator is much higher than that with only the PI controller. In addition to the frequency response with respect to the reference, Figure 5 shows the frequency response of the closed-loop system with respect to disturbances. As easily noticed, the gain for disturbance is relatively low in the whole interested frequency range. In other words, Figure 5 proves the robustness of the proposed compensator against the disturbance.

In Figure 2, the measured currents are directly used as feedback signals for the PI controller instead of the fundamental currents as described in the control laws (5) and (6) since the PI controller has no effect on high frequency signals. Furthermore, so far as the grid voltage is concerned, it is not necessary to separate the decoupling and feedforward terms as expressed in (5), (6), (10), and (11) for the fundamental and harmonic components since they are finally added up together. As a result, only one harmonic detector is needed as shown in Figure 2, which contributes to reduce significant computational efforts in the main controller. To extract the harmonic components from the output currents without phase delay, the fourth-order BPF has been implemented by cascading two second-order BPFs in the stationary reference frame. The transfer function of the second-order BPF can be described as 
$H_{B P F}(s)=\frac{K_{B P F} \beta s}{s^{2}+\beta s+\omega_{c}^{2}}$

where $\beta$ is the bandwidth, $\omega_{c}$ is the center frequency, and $K_{B P F}$ is the filter gain.

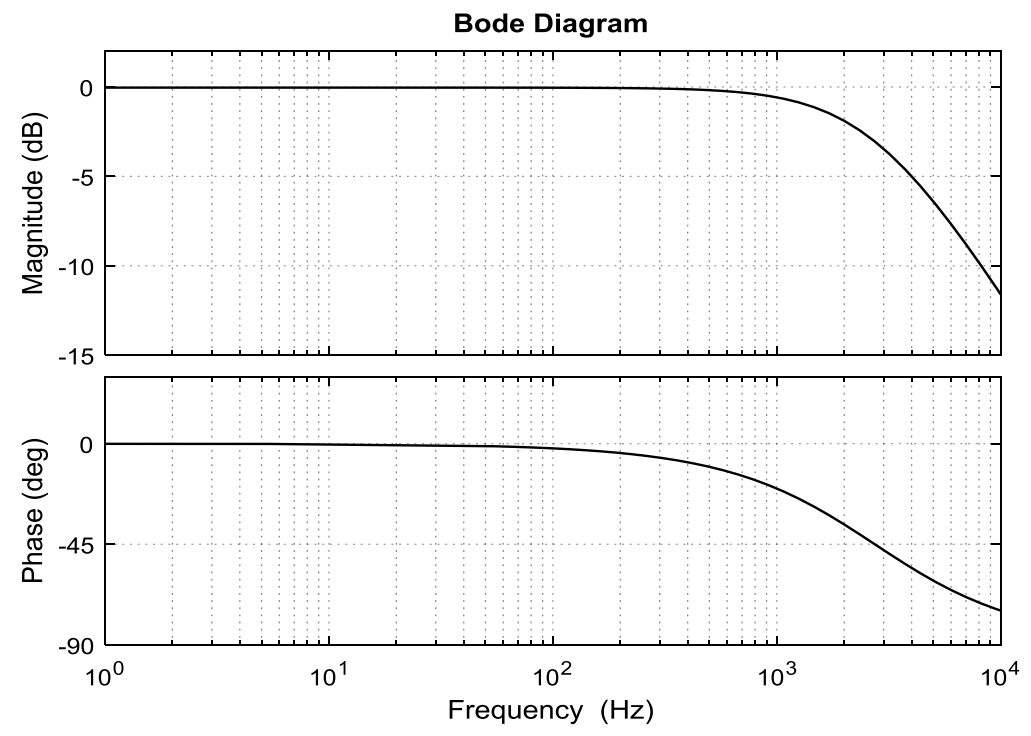

Figure 4. Frequency Response of a Grid-tied Inverter with the Proportional Harmonic Compensator

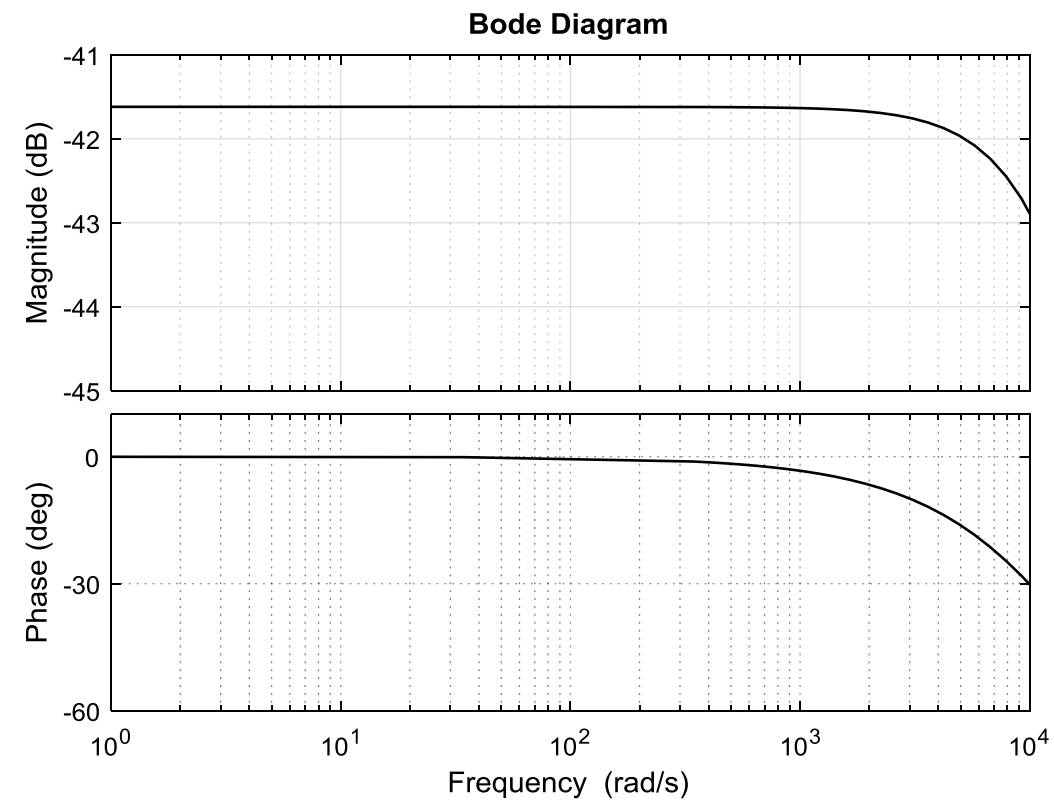

Figure 5. Frequency Response with Respect to Disturbances with the Proportional Harmonic Compensator 


\section{Simulation and Experimental Results}

To evaluate the performance of the proposed control scheme, the simulations and experiments have been carried out. The simulation model has been built using the PSIM simulation software based on the configuration as shown in Figure 1. Parameters which are used in the simulations and experiments are as follows: the DC-link voltage $V_{D C}=$ $420 \mathrm{~V}$, the inverter output filter resistance $R=0.5 \Omega$, and the inverter output inductance $L$ $=7 \mathrm{mH}$. In order to prove the performance of the proposed scheme in various operating conditions, the distorted grid voltage in the simulation model is formed by adding $10 \%$ of the fifth and the seventh harmonics and 5\% of the eleventh and the thirteenth harmonics to the sinusoidal grid voltages, as depicted in Figure 6.

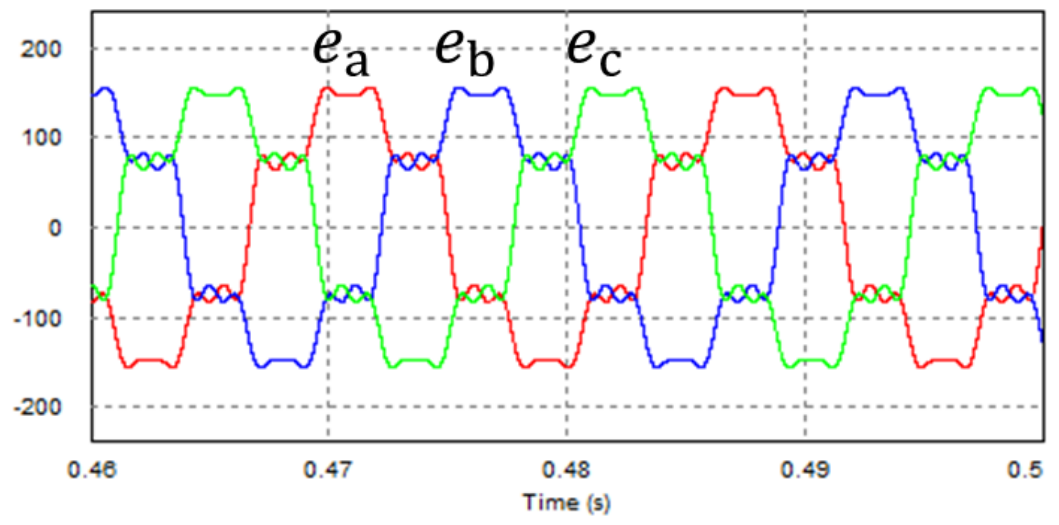

Figure 6. Three-phase Distorted Grid Voltages

The robustness of the proposed compensation scheme against the grid disturbances is demonstrated in Figure 7, where the steady-state response of the proposed control scheme is compared with the conventional PI decoupling controller. As is clearly shown, the inverter output currents of the conventional PI decoupling controller without compensation are highly distorted under the presence of the grid voltage harmonics. With the proposed compensation scheme, however, the inverter output currents remain fairly sinusoidal regardless of the highly distorted grid voltages as shown in Figure 7 (right).

In addition to the comparison in phase currents, Figure 8 and Figure 9 highlight the superior performance of the proposed control scheme by comparing the $q$-axis and $d$-axis currents in the synchronous reference frame under the same distorted grid voltage. As can be observed in these figures, the steady-state response of the convention PI decoupling controller reveals a quite high level of current ripple. By contrast, the reference currents are tracked with negligible current fluctuation in the proposed control scheme.
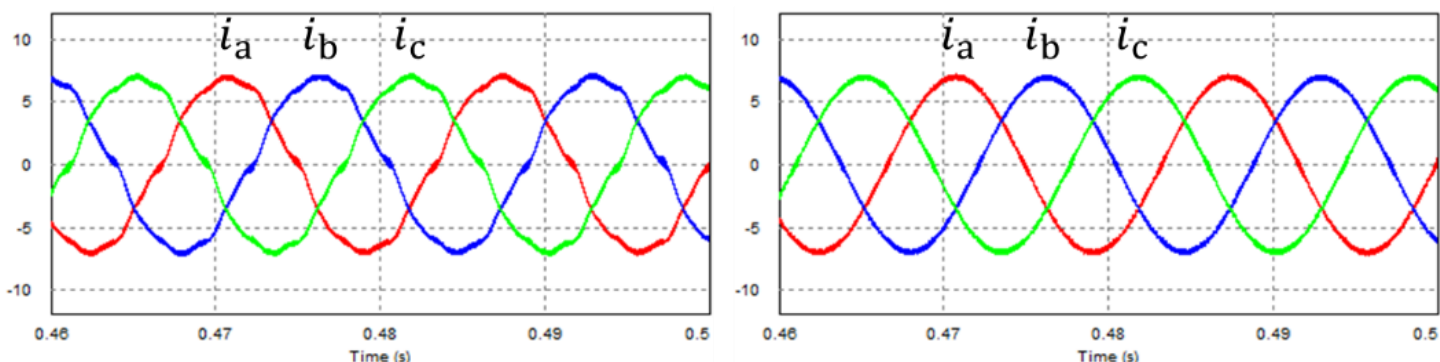

Figure 7. Inverter Output Currents under the Distorted Grid Voltages without Compensation (left) and with Compensation (right) 

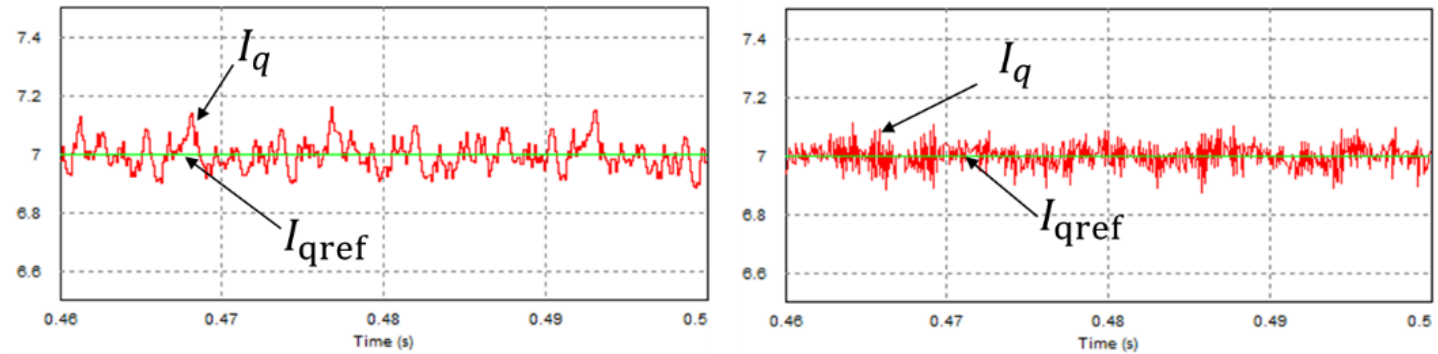

Figure 8. q-axis Inverter Output Currents under the Distorted Grid Voltages without Compensation (left) and with Compensation (right)
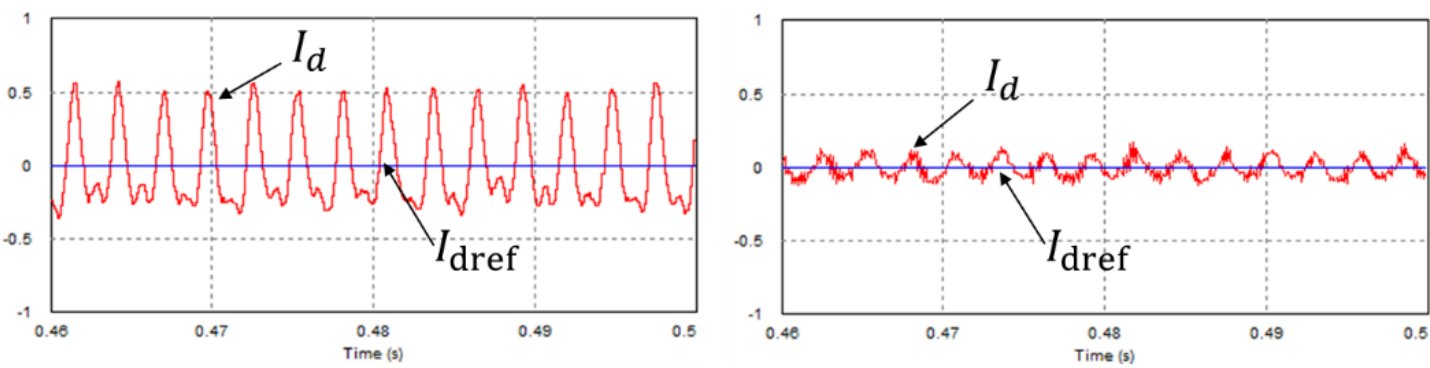

Figure 9. $d$-axis Inverter Output Currents under the Distorted Grid Voltage without Compensation (left) and with Compensation (right)

Along with steady-state responses, the transient responses of the proposed control scheme are given in Figure 10 and Figure 11. Figure 10 shows the transient response of the proposed control scheme for the step change in current reference. This figure reveals a relatively fast transient response as the $q$-axis current can approach the reference within around one period of grid voltage. On the other hand, Figure 11 illustrates the transient response of the proposed control scheme to disturbance. In this condition, the harmonics are suddenly superimposed on the ideal grid voltages at $0.3 \mathrm{~s}$, as shown in Figure 11(left). As a consequence of fast transient response of the proposed control scheme, the effect of disturbance grid voltage is instantly compensated, giving unnoticeable change in the current response as shown in Figure 11(right).

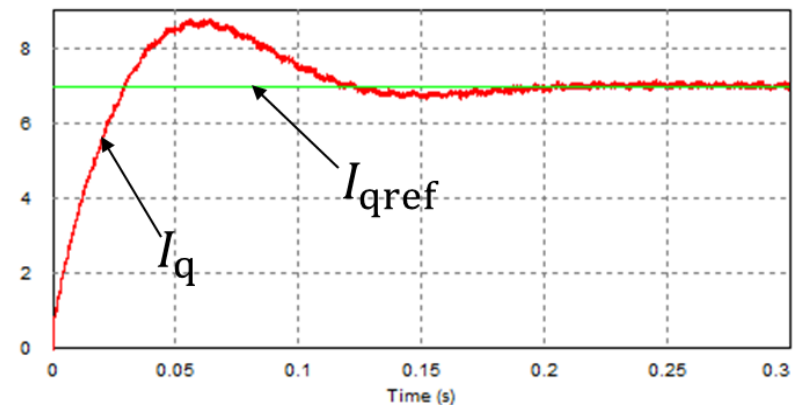

Figure 10. Step Response of $q$-axis Inverter Output Current 

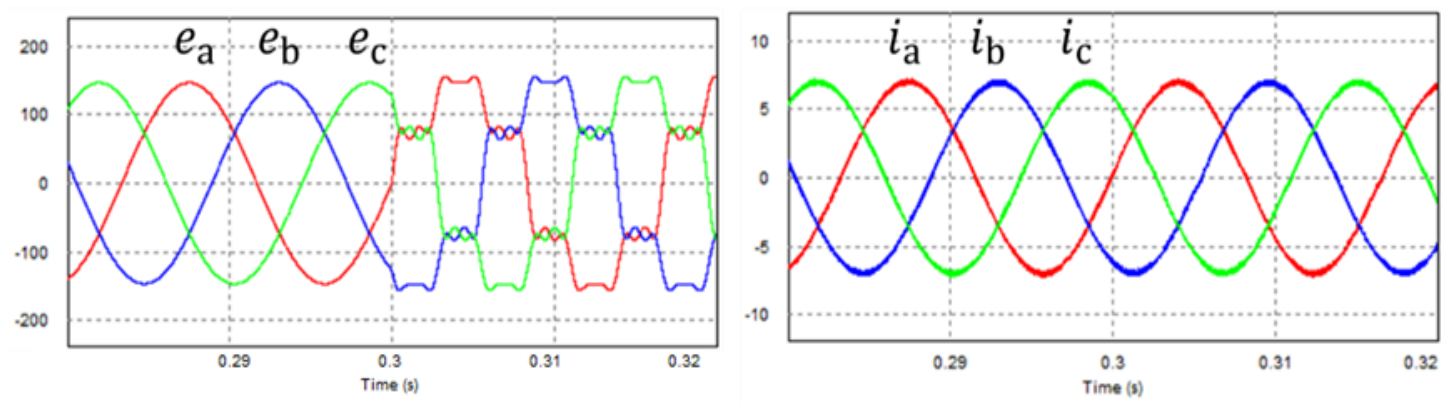

\section{Figure 11. Transient Responses of the Proposed Control Scheme, Three- phase Grid Voltages (left), Inverter Output Currents (right)}

For the experimental validation of the proposed control method, the experimental setup has been constructed, which consists of a DSP-based controller, an L-filtered threephase grid-tied inverter, a magnetic contactor for grid connecting operation, and a programmable AC power source to imitate the grid. The control algorithms are implemented by 32-bit floating-point DSP TMS320F28335 for 2 kVA laboratory prototype three-phase grid-tied inverter. Figure 12 shows three-phase distorted grid voltages used for experiments which include $10 \%$ of the fifth and the seventh harmonics and $5 \%$ of the eleventh and the thirteenth harmonics, resulting in the THD of $15.9 \%$.

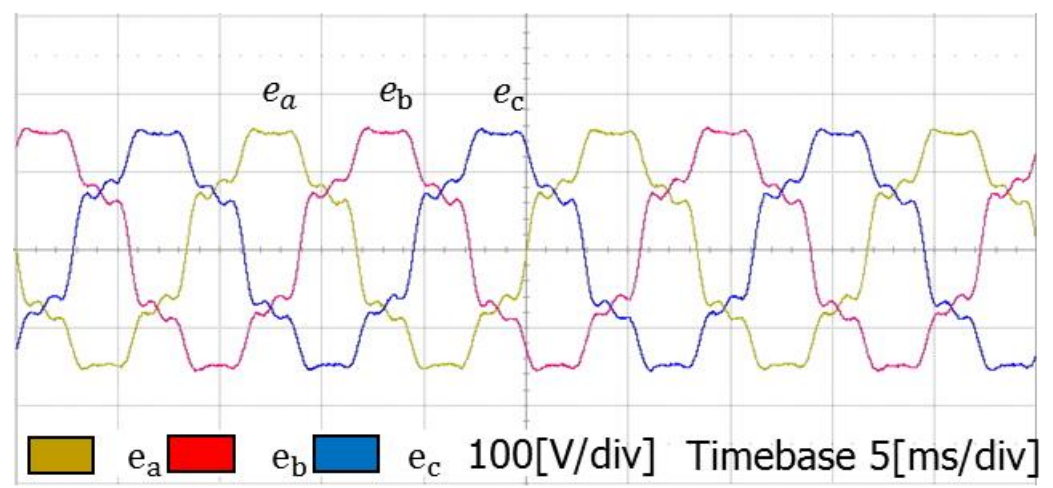

Figure 12. Three-phase Distorted Grid Voltages Used for Experiments

Figure 13 shows the experimental results for the inverter output current under the distorted grid voltage without compensation (left) and with compensation (right). The improvement of the proposed compensation scheme compared to the conventional controller can be clearly observed for the inverter output currents. This improvement is also illustrated in a sense of controlling the DC quantity of current in Figure 14, which shows the actual $q$-axis and $d$-axis inverter output currents in the steady-state together with their reference values. 

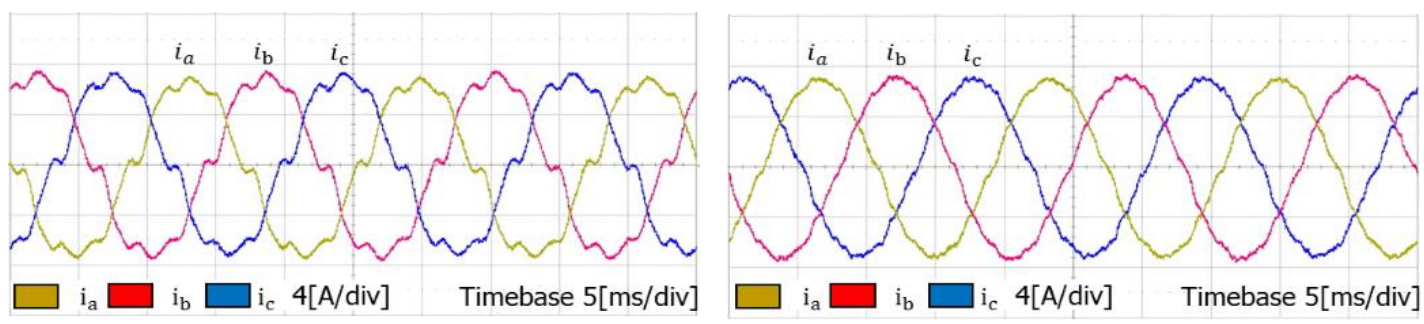

Figure 13. Experimental Results for Inverter Output Current under the Distorted Grid Voltage without Compensation (left) and with Compensation (right)
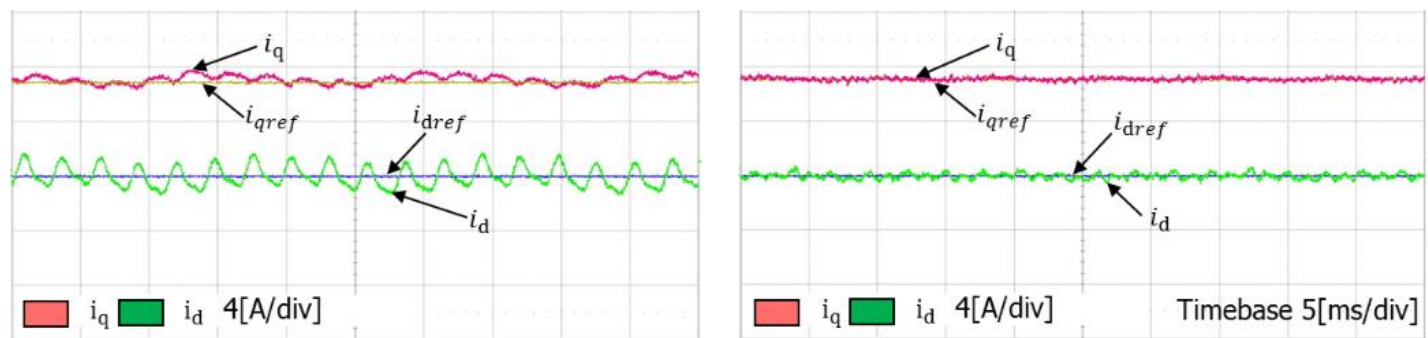

Figure 14. Experimental Results under the Distorted Grid Voltages for qaxis and d-axis Inverter Output Currents without Compensation (left) and with Compensation (right)

Figure 15 shows the FFT result of $a$-phase inverter output current for the proposed control scheme under the distorted grid voltage. As can be observed, the harmonic distortion level of phase current is spectacularly low for all harmonics in frequency range of interests. The THD level of inverter current in this extreme case is just over $2.5 \%$, which means that the THD level using the proposed control scheme is far less than the requirement of most integration codes.

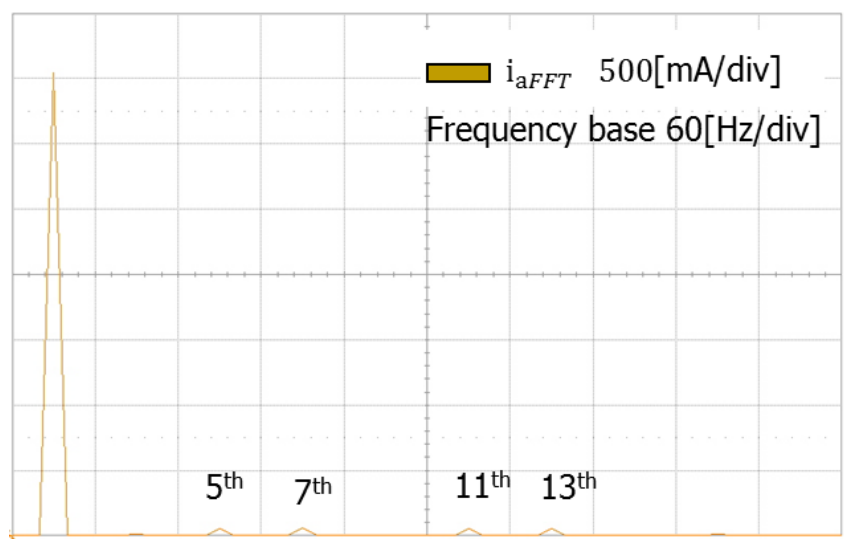

Figure 15. FFT Result of a-phase Current for the Proposed Scheme

The experimental outcomes presented from Figure 12 to Figure 15 confirm the simulation results as well as practical aspects of the proposed control scheme. Consequently, it is sufficient to deduce that the proposed scheme provides not only a good disturbance rejection capability but also a very fast transient response even in very simple way. 


\section{Conclusions}

To construct a reliable microgrid system, this paper has presented a simple harmonic mitigation strategy in a power electronic converter for the grid integration of renewable energy resources, which contributes to improve the power quality of grid-tied inverter in DG system in the presence of the distorted grid voltages. The proposed scheme divides the inverter currents into the fundamental and harmonic components by using the fourthorder digital BPF in order to control the fundamental and harmonic components of the inverter output currents separately. As a result of the separation, the harmonic currents can be instantly suppressed by proportional decoupling controller, while the fundamental current is stably controlled by the PI decoupling controller to inject the generated power to utility grid. Consequently, the proposed scheme ensures a fast dynamic response and provides a useful capability of reducing main harmful low order harmonics in three-phase system. The proposed scheme has a simple control structure with low computational burden. To prove the effectiveness of the proposed scheme, a prototype system has been constructed with a digital signal processor TMS320F28335. The comparative simulations and experimental results have demonstrated the performance of the proposed scheme as well as its simplicity. As a result, the proposed scheme can be one of the most effective ways to improve the performance of DG systems.

\section{Acknowledgements}

This study was supported by the Research Program funded by the Seoul National University of Science and Technology.

\section{References}

[1] M. Yazdanian and A. Mehrizi-Sani, "Distributed control techniques in microgrids", IEEE Transactions on Smart Grid, vol. 5, no. 6, (2014), pp. 2901-2909.

[2] Q. N. Trinh and H. H. Lee, "An advanced current control strategy for three-phase shunt active power filters", IEEE Transactions on Industrial Electronics, vol. 60, no. 12, (2013), pp. 5400-5410.

[3] R. Teodorescu, M. Liserre and P. Rodriguez, "Grid Converters for Photovoltaic and Wind Power Systems", John Wiley \& Sons Inc., (2011), pp. 314-320.

[4] M. Kazmierkowski, R. Krishnan and F. Blaabjerg, "Control in Power Electronics - Selected Problems", Academic Press, (2002).

[5] S. Guoqiao, Z. Xuancai, Z. Jun and X. Dehong, "A new feedback method for PR current control of LCL-filter-based grid-tied inverter", IEEE Transactions on Industrial Electronics, vol. 57, no. 6, (2010), pp. 2033-2041.

[6] M. Liserre, R. Teodorescu and F. Blaabjerg, "Multiple harmonics control for three phase grid converter systems with the use of PI-RES current controller in a rotating frame", IEEE Transactions on Power Electronics, (2006), pp. 836-841.

[7] H. S. Heo, G. H. Choe and H. S. Mok, "Robust predictive current control of a grid connected inverter with harmonics compensation", in Twenty-eighth Annual IEEE Applied Power Electronics Conference and Exposition (APEC), (2013), pp. 2212-2217.

[8] M. A. Abusara, M. Jamil and S. M. Sharkh, "Repetitive current control of an interleaved grid-tied inverter", Third IEEE International Symposium on Power Electronics for Distributed Generation Systems (PEDG), (2012), pp. 558-563.

[9] L. Tao, H. Xiang, Y. Xu, Z. Ming, H. Qingyun and H. Lang, "A novel repetitive control scheme for three-phase grid-tied inverter with LCL filter", Seventh International Power Electronics and Motion Control Conference (IPEMC), (2012), pp. 335-339.

[10] M. A. Abusara and S. M. Sharkh, "Design of a robust digital current controller for a grid con-nected interleaved inverter", IEEE international symposium on industrial electronics (ISIE), (2010), pp. 29032908.

[11] H. Xiang, Y. Xu, X. Ruiliang, H. Lang, L. Tao and L. Yang, "A fixed switching frequency in-tegral resonant sliding mode controller for three-phase grid-tied photovoltaic inverter with LCL-filter", IEEE ECCE Asia Conference (ECCE Asia), (2013), pp. 793-798.

[12] M. A. Sofla and R. King, "Control method for multi-microgrid systems in smart grid environment stability, optimization and smart demand participation”, IEEE PES Innovative Smart Grid Technologies (ISGT), (2012), pp. 1-5. 
[13] [S. L. Jung and Y. Y. Tzou, "Discrete sliding-mode control of a PWM inverter for sinusoidal output waveform synthesis with optimal sliding curve", IEEE Transactions on Power Electronics, (1996), pp. $567-577$.

[14] “TMS320F28335 Digital Signal Controller (DSC) - Data Manual”, Texas Instrument, (2008).

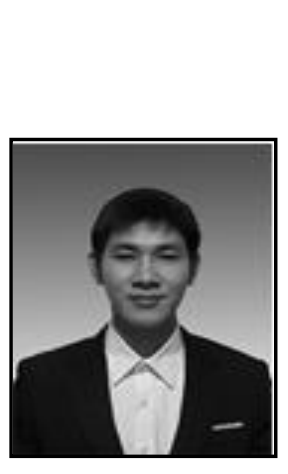

\begin{abstract}
Authors
Ngoc Bao Lai, he was born in Thanh Hoa, Vietnam, in 1991. He received the B.S. degree in electrical engineering from Danang University of Science and Technology, Da Nang, Vietnam, in 2014. $\mathrm{He}$ is currently working toward the M.S. degree in electrical and information engineering at Seoul National University of Science and Technology, Seoul, Korea. His current interests include robust control, power electronics, distributed generation systems, and microgrid. Mr. Lai is a student member of the Korean Institute of Power Electronics (KIPE) and IEEE.
\end{abstract}

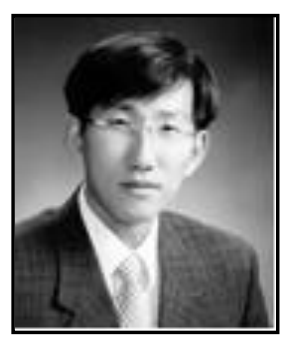

Kyeong-Hwa Kim, he was born in Seoul, Korea, in 1969. He received the B.S. degree from Hanyang University, Seoul, Korea, and the M.S. and Ph.D. degrees from Korea Advanced Institute of Science and Technology (KAIST), Taejon, Korea, in 1991, 1993, and 1998, respectively, all in electrical engineering. From 1998 to 2000, he was a Research Engineer with Samsung Electronics Company, where he was engaged in research and development of AC machine drive systems. From 2000 to 2002, he was a Research Professor with KAIST. From August 2010 to August 2011, he was a Visiting Scholar with Virginia Polytechnic Institute and State University (Virginia Tech), Virginia. Since August 2002, he has been with Seoul National University of Science and Technology, where he is currently a Professor. His current research interests are in the areas of AC machine drive, control, diagnosis, power electronics, renewable energy, and DSP-based control applications. Prof. Kim is a member of the Korean Institute of Power Electronics (KIPE) and IEEE. 
International Journal of Control and Automation

Vol. 10, No. 2 (2017) 